\title{
Spectroscopie résolue en temps par continuum femtoseconde Applications en neurobiologie
}

\author{
S. Ramstein et S. Mottin
}

Laboratoire TSI, UMR 5516 du CNRS, 42023 Saint-Etienne cedex 02, France

\begin{abstract}
Résumé : La spectroscopie résolue en temps utilisant un laser blanc femtoseconde est appliquée à la mesure in vivo des principaux absorbeurs du cerveau. Après génération ađéquate du continuum de lumière blanche femtoseconde $(50 \mathrm{~mW} /[580-756 \mathrm{~nm}]$ à $1 \mathrm{kHz})$, cette source se propage dans la calvaria, les méninges et le cortex chez le rat anesthésié. La transmission est étudiée sur $7 \mathrm{~mm}$ de distance entre l'impact laser et la fibre optique de collection. Le signal transmis est analysé dans la fenêtre $580-760 \mathrm{~nm}$, par un spectromètre couplé à une caméra à balayage de fente permettant la décorrélation de l'absorption et de la diffusion.
\end{abstract}

\section{INTRODUCTION}

Depuis les premières études optiques transcrâniennes chez les enfants hydrocéphales dans les années 60 [8], la lumière est utilisée pour étudier certaines fonctions cérébrales non accessibles aux techniques conventionnelles d'imagerie ou d'analyse fonctionnelle. La compréhension des couplages neurométaboliques et neurovasculaires sont la cible première de nos travaux. La fenêtre spectrale visible et proche IR permet de sonder la perfusion et l'oxygénation cérébrale grâce aux propriétés d'absorption des hémoglobines. Depuis dix ans des industriels (Hamamatsu, Siemens, General Electric, Hitachi, ...) ont développé de nombreux appareils. Cependant leurs choix technico-financiers n'ont pas permis la validation clinique: techniques continues, réponses impulsionnelles ou harmoniques à quelques longueurs d'onde. Afin de dépasser ces limites et de quantifier les principaux absorbeurs du cerveau de manière fiable, un nouveau dispositif à base de continuum a été développé [8]. Ce système de mesure de temps de vol large spectre est basé sur la génération d'un continuum de lumière blanche. La première observation du continuum est attribuée à Alfano et Shapiro [2]. Depuis, les applications en spectroscopie ont été nombreuses [10].

\section{MATERIEL ET METHODES}

\subsection{Le dispositif expérimental}

Le système laser est composé d'un oscillateur (Vitesse Coherent, $800 \mathrm{~nm}, 78 \mathrm{MHz}, 260 \mathrm{~mW}, 60 \mathrm{fs}$ ) et d'un amplificateur à dérive de fréquence (BMI/Thalès Alpha 1000). Les impulsions délivrées ont les caractéristiques suivantes : 800nm, 130-150fs (mesuré par autocorrélation, APE-Autocorrelator PulseCheck/PulseScope), $1 \mathrm{kHz}$, $500 \mathrm{~mW}$. En sortie du compresseur, le continuum est généré par focalisation du faisceau laser dans de l'eau pure déminéralisée. La focale de la lentille utilisée est de $21 \mathrm{~cm}$.

Le laser blanc obtenu est filtré spectralement (passe-bas, $\mathrm{T}>99 \% \quad \lambda<750 \mathrm{~nm}$ ) et spatialement (diaphragme circulaire). Le faisceau est amené sur le milieu d'étude grâce à un dispositif de trois miroirs en argent et une lentille de focale $10 \mathrm{~cm}$. Le point d'impact à la surface du crâne des animaux est fixé à $0.5 \mathrm{~mm}$ à angle droit pour les expériences présentées.

Après propagation dans les tissus (calvaria, méninges et couches corticales), la lumière transmise est collectée par une fibre optique de $0.2 \mathrm{~mm}$ de diamètre et $1.2 \mathrm{~m}$ de longueur. Le signal est analysé par un spectromètre (270M, Spex Jobin-Yvon) combiné à une caméra à balayage de fente (Streakscope, C4334, Hamamatsu). Les mesures sont effectuées en mode monocoup et par comptage de photoélectron unique 2D [8]. Le déclenchement sur chaque tir laser s'effectue à l'aide d'une photodiode rapide sensible (ET2000). Son utilisation en saturation permet de diminuer les effets de jitter dus au mode de declenchement monocoup de la caméra. Le dispositif autorise une analyse spectro-temporelle du signal sur une fenêtre spectrale de $176 \mathrm{~nm}$ avec une résolution temporelle de «2.4ps par pixel ». La réponse de la caméra sans déconvolution est de $14 \mathrm{ps}$ à mi-hauteur. Chaque image intègre la réponse sur $33 \mathrm{~ms}$ donc une accumulation de 33 coups laser.

La préparation animale est réalisée sous conditions stéréotaxiques. Les rats mâles OFA (300-400g, IFFA CREDO France) sont anesthésiés avec du chloral hydrate ( $400 \mathrm{mg} / \mathrm{kg}$, I.P.). Le crâne est mis à nu et nettoyé avec une solution saline. Les points d'impact et de coliection de la lumière sont positionnés à l'aide de l'atlas de 
Paxinos [9]. Les mesures in vivo (figure 2) ont été réalisées à la surface du crâne dans la longueur de l'hémisphère droit cérébral. La distance entre l'impact du laser blanc et la fibre de collection est fixée à $7 \mathrm{~mm}$. En fin d'expérimentation, les animaux sont sacrifiés par injection létale d'anesthésiant.

\subsection{Procédure de traitement des données}

L'approche mathématique utilisée est celle du transport radiatif de la lumière [3]. Dans cette approximation et en milieu semi-infini, la probabilité au temps $t$ de détecter un photon à la surface du milieu à une distance $\rho$ du point d'injection est donné par la formule de Patterson [6] :

$$
R(\rho, t)=U(t) \frac{1}{2}(4 \pi D)^{-z / 2} t^{-5 / 2} e^{-\frac{t}{\Sigma_{n}}} e^{-\frac{t_{F}(\rho, \underline{\underline{D}})}{t}}\left[z_{0} e^{-\frac{t_{F}\left(r_{1}, \underline{D}\right)}{t}}+z_{-1} e^{-\frac{\hat{t}\left(r_{1}, \underline{D}\right)}{t}}\right]
$$

Avec comme paramètres :

$$
\begin{gathered}
\mathrm{r}_{1}=\mathrm{z}_{0}=1 / \mu_{\mathrm{s}}{ }^{\prime} \quad \mathrm{r}_{-1}=\mathrm{z}_{\mathrm{o}}+2 \mathrm{z}_{\mathrm{b}} \quad \mathrm{z}_{\mathrm{b}}=2 \mathrm{D}\left(1+\underline{\mathrm{R}}_{\mathrm{d}}\right) /\left(1-\underline{\mathrm{R}}_{\mathrm{d}}\right) \\
\underline{\mathrm{R}}_{\mathrm{d}}=-1.440 \mathrm{n}^{-2}+0.710 \mathrm{n}^{-1}+0.0636 \mathrm{n} \\
\frac{1}{\sum_{\mathrm{a}}}=\mathrm{c} \mu_{\mathrm{a}} \quad \underline{\mathrm{D}}=\mathrm{cD} \quad \mathrm{D}=\frac{1}{3\left(\mu_{\mathrm{a}}+\mu_{\mathrm{s}}^{\prime}\right)} \quad \mathrm{t}_{\mathrm{F}}(\rho, \underline{\mathrm{D}})=\frac{\rho^{2}}{4\langle\underline{\mathrm{D}}\rangle}
\end{gathered}
$$

$\mu_{\mathrm{a}}$ et $\mu_{\mathrm{s}}$ ' sont les coefficients moyens d'absorption et de diffusion de la lumière. $r_{1}$ est le chemin effectif moyen, $r_{-1}$ la distance extrapolée, $U(t)$ la fonction de Heaviside et $t_{F}$ le temps de Fourier.

$\mathrm{La}$ fonction de réponse instrumentale $h(t)$ n'étant pas un dirac temporel, le signal est la convolution :

$$
S(t)=\int_{-\infty}^{+\infty} R(\tau) h(t-\tau) d \tau
$$

Le signal mesuré est comparé à $\mathrm{S}(\mathrm{t})$ par la méthode des moindres carrés.

\section{RESULTATS}

Les mesures spectro-temporelles (figures 1 et 2) sont effectuées par intégration de 25000 coups laser à $1 \mathrm{kHz}$ (temps de mesure $=25 \mathrm{~s}$ ). Elles sont enregistrées de manière à avoir un taux de comptage comparable dans la région $730-750 \mathrm{~nm}$.

\subsection{Réponse instrumentale (IRF)}

Le spectre du continuum généré dans l'eau couvre une large fenêtre : 380 à $900 \mathrm{~nm}$ [8]. La puissance sur $400 \mathrm{~nm}$ $(380 \mathrm{~nm}-780 \mathrm{~nm}$ ) est de l'ordre de $100 \mathrm{~mW}$ pour une puissance incidente de $500 \mathrm{~mW}$ à $800 \mathrm{~nm}$. Le rendement correspondant est $I[380 \mathrm{~nm}-780 \mathrm{~nm}] /[$ [ $800 \mathrm{~nm}]=20 \%$. Les mesures in vivo sont effectuées avec une puissance de l'ordre de $50 \mathrm{~mW}$ sur la fenêtre spectrale d'intérêt $(580-760 \mathrm{~nm})$.

L'image spectro-temporelle de la figure 1 est un exemple de fonction de réponse instrumentale non corrigée de l'ensemble du dispositif (pas de filtre pour aplatir le spectre, pas de correction de la sensibilité spectrale de la photocathode). La largeur à mi hauteur du signal est de $24 \mathrm{ps}$ entre 670 et $695 \mathrm{~nm}$ (figure 6).

\subsection{Mesures in vivo}

La figure 2 est un exemple de mesure de la propagation du continuum par transillumination crânienne chez le rat anesthésié. On peut remarquer en particulier l'élargissement de l'impulsion causé par la diffusion et l'absorption très marquée en dessous de $650 \mathrm{~nm}$. Les spectres d'absorption des hémoglobines considérées comme les principaux absorbeurs dans cette fenêtre sont présentés sur la figure 3. Le coefficient d'absorption est donné par rapport à un globule rouge $\left(R B C, V_{R B C}=61 \mu \mathrm{m}^{3}\right.$ chez le rat). En effet l'absorption des tissus n'est localisée qu'à leur niveau. Sur le trajet de son diamètre moyen un $\mathrm{RBC}$ absorbe $10 \%$ de la lumière à $600 \mathrm{~nm}$ $\left(\mu_{\mathrm{n}}(\lambda)_{\mathrm{RBC}}=2.3 \varepsilon(\lambda)[\mathrm{Hb}, \mathrm{HbO} 2]_{\mathrm{RBC}}\right.$ avec $\left.[\mathrm{Hb}, \mathrm{HbO} 2]_{\mathrm{RBC}}=4.332 \mathrm{mmol.} \mathrm{L}^{-1}\right)$. 

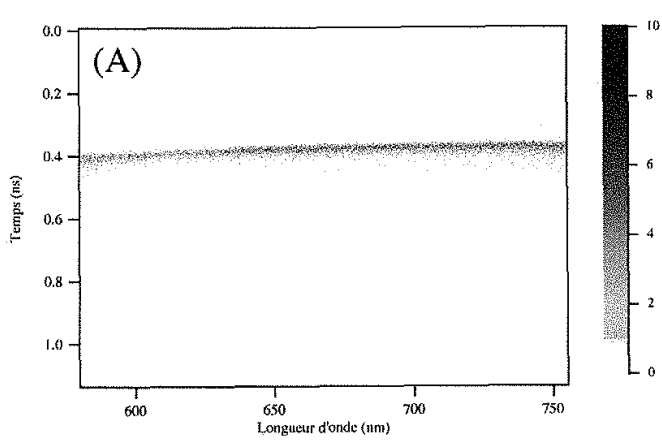

Figure 1. Image spectro-temporelle de la fonction de réponse instrumentale. Intégration sur 25s. (SPE: Single Photo-Electron).

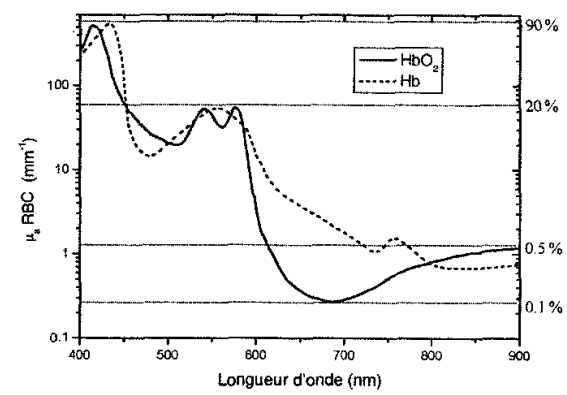

Figure 3. Spectre d'absorption des hémoglobines pour un globule rouge $(\mathrm{RBC})$. Les pourcentages de lumière absorbée pour un seul RBC sont indiqués.
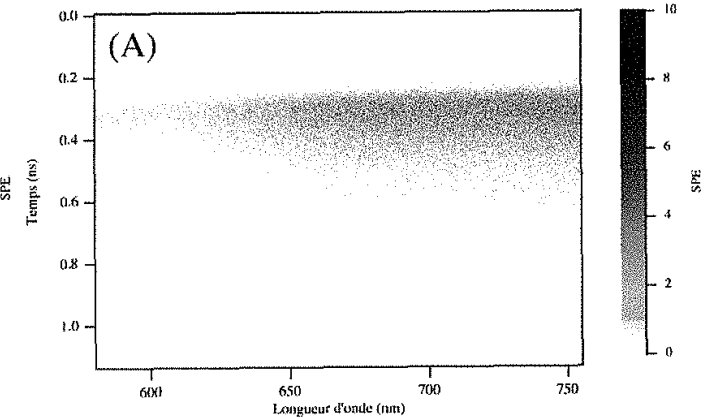

Figure 2. Image de spectroscopie résolue en temps. La distance point source/fibre de collection est de $7 \mathrm{~mm}$. Intégration sur $25 \mathrm{~s}$.

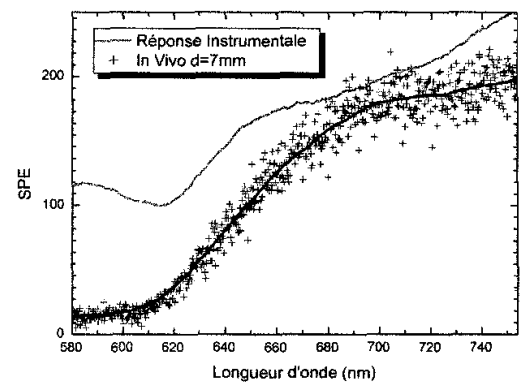

Figure 4.. Spectres de transmisssion. Intégration temporelle des images 1 et 2 .

La figure 4 présente les différences spectrales sur la fenêtre 580-760nm entre l'IRF et la mesure in vivo. Ces spectres de transmission sont obtenus en intégrant temporellement les images des figures 1 et 2 .

Le spectre brut d'absorption présenté sur la figure 5 est obtenu avec l'approximation que le log du ratio de la mesure in vivo/IRF est proche de zéro pour la zone autour de $740 \mathrm{~nm}$, fenêtre spectrale où la somme des hémoglobines absorbe le moins. La pente entre 620 et $740 \mathrm{~nm}$ est très importante. La figure 6 illustre la comparaison entre les profils temporels de l'IRF et d'une mesure in vivo pour la fenêtre spectrale 670-695nm. L'analyse à l'aide de la fonction de Patterson permet d'obtenir les coefficients d'absorption et de diffusion. Par exemple dans la fenêtre spectrale $670-695 \mathrm{~nm}$, les coefficients d'absorption $\left\langle\mu_{\mathrm{a}}\right\rangle$ et de diffusion $\left\langle\mu_{\mathrm{s}}{ }^{\prime}\right\rangle$ sont respectivement de $0.054 \mathrm{~mm}^{-1}$ et de $2.45 \mathrm{~mm}^{-1}$.

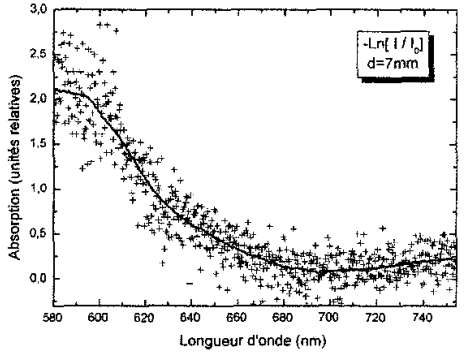

Figure 5. Spectre d'absorption obtenu en considérant que $\operatorname{Ln}\left[\mathrm{l} / \mathrm{l}_{0}\right]$ est proche de 0 autour de $740 \mathrm{~nm}$.

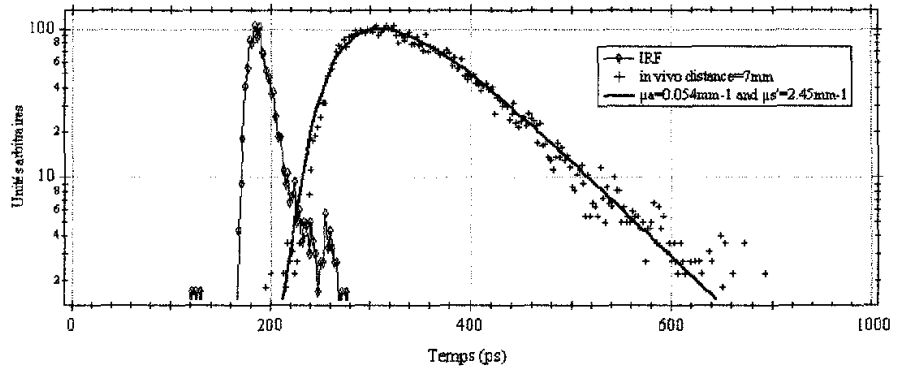

Figure 6. Comparaison des profils temporels de la réponse instrumentale et d'une mesure in vivo pour une fenêtre spectrale $670 \mathrm{~nm}-695 \mathrm{~nm}$. 


\section{DISCUSSION ET PERSPECTIVES}

En dessous de $620 \mathrm{~nm}$ et pour une distance de $7 \mathrm{~mm}$, l'absorption des tissus est trop importante pour ce dispositif. Ainsi les images de spectroscopie résolue en temps présentent dans cette plage peu de photoélectrons comptés. Dans cette gamme de longueurs d'onde $(<620 \mathrm{~nm})$, les hémoglobines absorbent très fortement (figure 3). Malgré une précompression optimisée, le rendement de conversion de lumière blanche $(800 \mathrm{~nm} \rightarrow 580-760 \mathrm{~nm})$ reste faible pour notre dispositif (environ 10\%). Des améliorations sont envisagées pour obtenir une puissance du laser blanc supérieure à $200 \mathrm{~mW}$. Le rendement dépend notamment des propriétés spatiales et temporelles du faisceau au site de génération du continuum ainsi que de la nature du milieu utilisé [5]. Nous espérons améliorer ce rendement par une mise en forme spatiale du faisceau laser plus optimisée. L'ajout de sels adaptés permettent aussi un meilleur taux de conversion [1].

Les coefficients d'absorption et de diffusion obtenus pour la mesure in vivo sont dans la gamme habituelle. Par exemple, dans le cas du cerveau de porc en post-mortem à $670 \mathrm{~nm}$ [3], ils sont de $3.1 \pm 0.2 \mathrm{~mm}^{-1}$ pour le $\mu_{\mathrm{s}}$ ' et $0.007 \pm 0.001 \mathrm{~mm}^{-1}$ pour le $\mu_{\mathrm{a}}$.

Pour calculer les coefficients moyens, les absorbeurs sont supposés distribués de manière totalement diluée dans les tissus. Hors l'absorption est surtout localisée dans les RBC limités au compartiment vasculaire. La compartimentation des hémoglobines reste un axe de recherche actuel [4-7-12]. Afin d'explorer cet effet sur nos mesures in vivo, un modèle périodique de vascularisation corticale chez le rat a été développé [11]. La théorie de l'homogénéisation sera appliquée pour réaliser la déconvolution fiable des coefficients d'absorption et de diffusion en fonction de la longueur d'onde dans ces structures aux propriétés optiques complexes.

\section{CONCLUSION}

A notre connaissance, nous présentons le premier système de spectroscopie kilohertz résolue en temps par laser blanc femtoseconde en particulier pour la neurophotonique in vivo. Les avantages de la technique et du système permettent un large domaine d'accordabilite (de l'ordre de $500 \mathrm{~nm}$ ). Sur un temps d'intégration de $25 \mathrm{~s}$, la réponse instrumentale offre une résolution temporelle brute de $24 \mathrm{ps}$ autorisant une mesure des temps de vol des photons dans l'eau de l'ordre de $3-4 \mathrm{~mm}$. Il permet la mesure simultanée et en temps réel des coefficients d'absorption et de diffusion des tissus sur un large spectre continu. Ici les résultats sur la fenêtre spectrale 670$695 \mathrm{~nm}$ se situent dans la gamme des mesures usuelles avec des sources à $670 \mathrm{~nm}$.

\section{Remerciements}

Les auteurs souhaitent remercier Pr. Raymond Cespuglio et Dr. Nicolas Mathevon pour leur aide.

\section{Rëférences}

[1] Alfano R. R., The supercontinuum laser source. (Springer-Verlag, New-York, 1989).

[2] Alfano R. R. et al., "Observation of self-phase modulation and small-scale filaments in crystals and glasses.", Phys. Rev. Let. 24 (1970) 592-594.

[3] Avrillier S. et al. "Propagation d'impulsions ultracourtes dans les milieux diffusants. Application au diagnostic medical." Systèmes ferntosecondes. (Laporte P., Salin F. et Mottin S., Publications de l'université de Saint-Etienne, SaintEtienne, 2001) 295-310.

[4] Bradu A. et al. "In-vivo absorption spectroscopy in brain using small optical fiber probes: effect of blood confinement." Proceedings of SPIE: Diagnostic optical spectroscopy in biomedicine. (Theodore G., Papazoglou et Wagnieres G. A., 2001) 4432 85-90.

[5] Brodeur A. et al., "Ultrafast white-light continuum generation and self-focusing in transparent condensed media.", J. Opt. Soc. Am. B 16 (1999) 637-650.

[6] Kienle A. et al., "Improved solutions of the steady state and the time-resolved diffusion equations for reflectance from a semi-infinite turbid medium.", J. Opt. Soc. Am. A 14 (1997) 246-254.

[7] Liu H. et al., "Influence of blood vessels on the measurement of hemoglobin oxygenation as determined by timeresolved reflectance spectroscopy.", Med. Phys. 22 (1995) 1209-1217.

[8] Mottin S. et al., "Génération d'un continuum de lumière blanche pour l'analyse spectrale résolue en temps de milieux très diffusants.", J. Phys. IV 11 (2001) 185-186.

[9] Paxinos G. et al., The rat Brain in stereotaxic coordinates Third Edition. (Academic Press, New York, 1997).

[10] Pommeret S. et al. "Artefacts dans une expérience pompe-sonde." Systèmes femtosecondes. (Laporte P., Salin F. et Mottin S., Publications de l'université de Saint-Etienne, Saint-Etienne, 2001) 183-207.

[11] Ramstein S. et al. "In vivo spectroscopy with femtosecond white light continuum." Proceedings of SPIE: Optical biopsy IV. (Alfano R. R., 2002) 4613 188-197.

[12] Van Veen R. L. P. et al. "Correction for inhomogeneously distributed absorbers in spatially resolved diffuse reflectance spectroscopy." Proceedings of SPIE: Photon migration, optical coherence tomography, and microscopy. (Andersson-Engels S. et Kaschke M. F., 2001) 4431 192-194. 